\title{
Azimuthal Asymmetries for eA/eN Semi-Inclusive DIS and Its Nuclear Dependence
}

\author{
Yu-Kun Song \\ School of Physics and Technology, University of Jinan, Jinan, Shandong 250022, China \\ sps_songyk@ujn.edu.cn
}

Published 29 February 2016

\begin{abstract}
We applied collinear expansion to the semi-inclusive deeply inelastic lepton-nucleon (nucleus) scattering process $e+N(A) \rightarrow e+q+X$ with both polarized beam and polarized target up to twist-3, and unpolarized process up to twist- 4 . The differential cross section and azimuthal asymmetries are expressed in terms of gauge invariant twist-3 and twist-4 TMD parton distribution/correlation functions. Measurements of such azimuthal asymmetries provide methods to study different spin and transverse momentum aspects of the partonic structure of nucleon. We further study the nuclear dependence of azimuthal asymmetries and adopt Gaussian ansatz for TMD distribution/correlation functions to estimat the semi-quantitive behaviour of the nuclear dependence. We predict the Adependence of azimuthal asymmetries which can be tested in the planned EIC's.
\end{abstract}

Keywords: Collinear expansion; SIDIS; Azimuthal asymmetry.

\section{Introduction}

Semi-inclusive deeply inelastic lepton-nucleon scattering (SIDIS) provides a nice place to study the spin- and transverse-momentum-dependent(TMD) parton distribution functions. Measurements of azimuthal asymmetries of final state hadron(jet) of SIDIS has been done in Hermes, COMPASS and JLab, and they are proposed to be important observables for the planned electron-ion colliders (EIC's). However, most quantitative theoretical analysis of azimuthal asymmetries are limited to leading twist level, ignoring higher twist contributions which might give large contributions at currently available experimental energy and might generates new azimuthal dependences. Incorporation of higher twist contributions is an urgent task for theorist, but there is a lot of mists ahead to be cleared. The fundamental QCD TMD factorization at twist-3 or higher twist level is not proved yet, and very little quantitative informaiton of higher twist parton correlation functions is extracted from experimental data.

This is an Open Access article published by World Scientific Publishing Company. It is distributed under the terms of the Creative Commons Attribution 3.0 (CC-BY) License. Further distribution of this work is permitted, provided the original work is properly cited. 
Recently we have focused on the theoretical framework of higher twists in SIDIS, and propose methods to relate experimental observables with gauge invariant matrix elements. We adopt collinear expansion in inclusive DIS ${ }^{1-3}$ and applied this technique to SIDIS. ${ }^{4-8}$ We obatin gauge invariant hadronic tensors and differential cross sections. The azimuthal asymmetries are obtained as proportional to the ratios of different TMD parton distribution/correlation functions, and they might significantly deviate from Cahn's parton model results. ${ }^{9}$ The measurements of various azimuthal asymmetries provide a direct way of studying higher twist TMD parton correlation functions. We studied SIDIS up to twist-3 for various polarized scenarios for initial states, ${ }^{7}$ and up to twist- 4 for the unpolarized case. ${ }^{6}$ We further studied the nuclear effects on azimuthal asymmetries if one replace the initial state nucleon with a large nucleus, and find interesting A-dependences. ${ }^{8}$ In the next few sections I will summarize the theoretical frameworks, the results for differential cross sections and azimuthal asymmetries, and the nuclear modifications of azimuthal asymmetries.

\section{Collinear Expansion Applied to SIDIS}

The differential cross section of SIDIS can be expressed as,

$$
d \sigma=\frac{2 \alpha_{\mathrm{em}}^{2} e_{q}^{2}}{s Q^{4}} L^{\mu \nu}\left(l, l^{\prime}, s_{l}\right) \frac{d^{2} W_{\mu \nu}}{d^{2} k_{\perp}^{\prime}} \frac{d^{3} l^{\prime} d^{2} k_{\perp}^{\prime}}{2 E_{l^{\prime}}},
$$

where $\frac{d^{2} W_{\mu \nu}}{d^{2} k_{\perp}^{\prime}}$ is the hadonic tensor which include all information of strong interaction and nucleon structure. Taking QCD interaction into consideration there are mulitple gluon interactions that contribute and the hadronic tensor is the sum of a tower of Feynman diagrams, as shown in Fig. 1. Each diagram is expressed as the product of the hard part and the parton correlation matrix,

$$
\begin{aligned}
W_{\mu \nu}^{(0, \mathrm{si})}= & \frac{1}{2 \pi} \int \frac{d^{4} k}{(2 \pi)^{4}} \operatorname{Tr}\left[\hat{H}_{\mu \nu}^{(0)}(k, q) \hat{\phi}^{(0)}(k, p, S)\right] 2 E_{k^{\prime}}(2 \pi)^{3} \delta^{3}\left(\vec{k}^{\prime}-\vec{k}-\vec{q}\right), \\
W_{\mu \nu}^{(1, \mathrm{si})}= & \frac{1}{2 \pi} \int \frac{d^{4} k_{1}}{(2 \pi)^{4}} \frac{d^{4} k_{2}}{(2 \pi)^{4}} \sum_{c=L, R} 2 E_{k^{\prime}}(2 \pi)^{3} \delta^{3}\left(\vec{k}^{\prime}-\vec{k}_{c}-\vec{q}\right) \\
& \times \operatorname{Tr}\left[\hat{H}_{\mu \nu}^{(1, c) \rho}\left(k_{1}, k_{2}, q\right) \hat{\phi}_{\rho}^{(1)}\left(k_{1}, k_{2}, p\right)\right],
\end{aligned}
$$

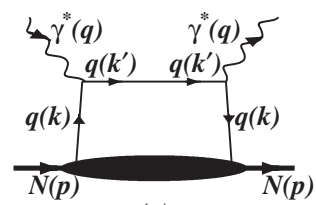

(a)

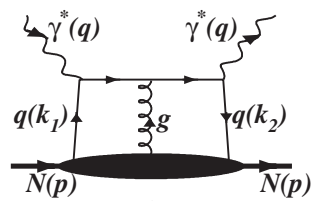

(b)

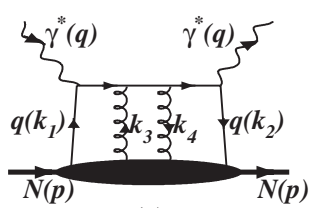

(c)

Fig. 1. Feynman diagrams for SIDIS with multiple gluon exchange. 
where $c$ denotes the position of the cut line, $k_{L}=k_{1}, k_{R}=k_{2}, k_{M}=k$, and the hard parts are given by,

$$
\begin{aligned}
& \hat{H}_{\mu \nu}^{(0)}(q, k)=\gamma_{\mu}(\not k+\not q) \gamma_{\nu}(2 \pi) \delta_{+}\left((k-q)^{2}\right), \\
& \hat{H}_{\mu \nu}^{(1, L) \rho}\left(k_{1}, k_{2}, q\right)=\gamma_{\mu}\left(k_{2}+\not q\right) \gamma^{\rho} \frac{\not k_{1}+\not}{\left(k_{1}+q\right)^{2}-i \epsilon} \gamma_{\nu}(2 \pi) \delta_{+}\left(\left(k_{2}+q\right)^{2}\right),
\end{aligned}
$$

and the matrix elements or the correlators are defined as,

$$
\begin{aligned}
& \hat{\phi}^{(0)}(k, p, S)=\int d^{4} z e^{i k z}\langle p, S|\bar{\psi}(0) \psi(z)| p, S\rangle, \\
& \hat{\phi}_{\rho}^{(1)}\left(k_{1}, k_{2}, p, S\right)=\int d^{4} y d^{4} z e^{i k_{1} z+i\left(k_{2}-k_{1}\right) y}\left\langle p, S\left|\bar{\psi}(0) g A_{\rho}(z) \psi(y)\right| p, S\right\rangle .
\end{aligned}
$$

As can be seen from above definition for $\hat{\phi}^{(i)}$, since each field lie on different spacetime points, under local color gauge transformation they are not invariant. This means that these matrices are not physical observables and one must proceed to render them gauge invariant.

The standard way of obtaning gauge invariant matrix elements is collinear expansion, which involves following 4 steps:

(1) Taylor expansion of hard parts,

$$
\hat{H}_{\mu \nu}^{(0)}(k)=\hat{H}^{(0)}(x)+\frac{\partial \hat{H}^{(0)}(x)}{\partial k^{\rho}} \omega^{\rho}{ }_{\rho^{\prime}} k^{\rho^{\prime}}+\frac{1}{2} \frac{\partial^{2} \hat{H}^{(0)}(x)}{\partial k^{\rho} \partial k^{\sigma}} \omega_{\rho^{\prime}} k^{\rho^{\prime}} \omega^{\sigma}{ }_{\sigma^{\prime}} k^{\sigma^{\prime}}+\ldots
$$

(2) Decomposition of gluon field into collinear and non-collinear parts,

$$
A^{\rho}=\frac{A^{+}}{p^{+}} p^{\rho}+\omega_{\rho^{\prime}}^{\rho} A^{\rho^{\prime}},
$$

(3) Use Ward identities to relate different hard parts.

$$
\frac{\partial \hat{H}_{\mu \nu}^{(0)}(x)}{\partial k^{\rho}}=-\sum_{c=L, R} \hat{H}_{\mu \nu}^{(1, c) \rho}(x, x), \quad p_{\rho} \hat{H}^{(1, L) \rho}\left(x_{1}, x_{2}\right)=\frac{\hat{H}_{\mu \nu}^{(0)}\left(x_{1}\right)}{x_{2}-x_{1}-i \epsilon} .
$$

(4) Sum up and rearrange all terms to obtain gauge invariant correlation matrices.

$$
\begin{aligned}
\frac{d^{2} W_{\mu \nu}}{d^{2} k_{\perp}^{\prime}}= & \frac{d^{2} \tilde{W}_{\mu \nu}^{(0)}}{d^{2} k_{\perp}^{\prime}}+\frac{d^{2} \tilde{W}_{\mu \nu}^{(1)}}{d^{2} k_{\perp}^{\prime}}+\ldots, \\
\frac{d \tilde{W}_{\mu \nu}^{(0)}}{d^{2} k_{\perp}^{\prime}}= & \frac{1}{2 \pi} \int d x d^{2} k_{\perp} \operatorname{Tr}\left[\hat{H}_{\mu \nu}^{(0)}(x) \hat{\Phi}^{(0) N}\left(x, k_{\perp}\right)\right] \delta^{(2)}\left(\vec{k}_{\perp}-{\overrightarrow{k^{\prime}}}_{\perp}\right), \\
\frac{d \tilde{W}_{\mu \nu}^{(1)}}{d^{2} k_{\perp}^{\prime}}= & \frac{1}{2 \pi} \int d x_{1} d^{2} k_{1 \perp} d x_{2} d^{2} k_{2 \perp} \\
& \times \sum_{c=L, R} \operatorname{Tr}\left[\hat{H}_{\mu \nu}^{(1, c) \rho}\left(x_{1}, x_{2}\right) \omega_{\rho}^{\rho^{\prime}} \hat{\Phi}_{\rho^{\prime}}^{(1) N}\left(x_{1}, k_{1 \perp}, x_{2}, k_{2 \perp}\right)\right] \delta^{(2)}\left(\vec{k}_{c \perp}-\overrightarrow{k^{\prime}} \perp\right),
\end{aligned}
$$


where the tilded symbols $\tilde{W}^{(j)}$ 's represent results after collinear expansion and $\omega_{\rho}^{\rho^{\prime}}=g_{\rho}^{\rho^{\prime}}-\bar{n}_{\rho} n^{\rho^{\prime}}$ is a projection operator. The parton correlation matrices take the gauge invariant form and are given by,

$$
\begin{aligned}
& \hat{\Phi}^{(0) N}\left(x, k_{\perp}\right)=\int \frac{p^{+} d y^{-} d^{2} y_{\perp}}{(2 \pi)^{3}} e^{i x p^{+} y^{-}-i \vec{k}_{\perp} \cdot \vec{y}_{\perp}}\langle N|\bar{\psi}(0) \mathcal{L}(0 ; y) \psi(y)| N\rangle, \\
& \hat{\Phi}_{\rho}^{(1) N}\left(x_{1}, k_{1 \perp}, x_{2}, k_{2 \perp}\right)= \int \frac{p^{+} d y^{-} d^{2} y_{\perp}}{(2 \pi)^{3}} \frac{p^{+} d z^{-} d^{2} z_{\perp}}{(2 \pi)^{3}} \\
& \times e^{i x_{2} p^{+} z^{-}-i \vec{k}_{2 \perp} \cdot \vec{z}_{\perp}+i x_{1} p^{+}\left(y^{-}-z^{-}\right)-i \vec{k}_{1 \perp} \cdot\left(\vec{y}_{\perp}-\vec{z}_{\perp}\right)} \\
& \times\left\langle N\left|\bar{\psi}(0) \mathcal{L}(0 ; z) D_{\rho}(z) \mathcal{L}(z ; y) \psi(y)\right| N\right\rangle,
\end{aligned}
$$

where $\mathcal{L}(0 ; y)$ is the gauge link obtained in the collinear expansion, which generate extra factor under local gauge transformation and render the correlation matrices gauge invariant.

The rest of the formalism contain just routine procedures. One just need to expand the gauge invariant correlation matrices with $\gamma$-matrices to obtain the TMD parton distribution/correlation functions, and employ QCD equation of motion to simplify the final results for the hadronic tensor, which will be electromagnetic and color gauge invariant. After contraction with the leptonic tensor one obtain the cross section. For more details, see Refs. 4, 6 .

\section{Cross Section and Azimuthal Asymmetries in SIDIS}

The cross section up to twist-3 polarized SIDIS $e^{-}\left(l, s_{l}\right)+N(p, s) \rightarrow e^{-}\left(l^{\prime}\right)+q\left(k^{\prime}\right)+$ $X$ is, ${ }^{7}$

$$
\frac{d \sigma}{d x_{B} d y d^{2} k_{\perp}}=\frac{2 \pi \alpha_{\mathrm{em}}^{2} e_{q}^{2}}{Q^{2} y}\left(W_{U U}+\lambda_{l} W_{L U}+s_{\perp} W_{U T}+\lambda W_{U L}+\lambda_{l} \lambda W_{L L}+\lambda_{l} s_{\perp} W_{L T}\right)
$$

$$
W_{U U}=A(y) f_{1}-\frac{2 x_{B}\left|\vec{k}_{\perp}\right|}{Q} B(y) f^{\perp} \cos \phi,
$$

$$
\begin{aligned}
W_{U T}= & \frac{\left|\vec{k}_{\perp}\right|}{M} A(y) f_{1 T}^{\perp} \sin \left(\phi-\phi_{s}\right) \\
& -\frac{2 x_{B} M}{Q} B(y)\left[f_{T} \sin \phi_{s}-\frac{k_{\perp}^{2}}{2 M^{2}} f_{T}^{\perp} \sin \left(2 \phi-\phi_{s}\right)\right],
\end{aligned}
$$

$W_{U L}=-\frac{2 x_{B}\left|\vec{k}_{\perp}\right|}{Q} B(y) f_{L}^{\perp} \sin \phi$,

$$
W_{L U}=-\frac{2 x_{B}\left|\vec{k}_{\perp}\right|}{Q} D(y) g^{\perp} \sin \phi
$$

$$
W_{L L}=C(y) g_{1 L}-\frac{2 x_{B}\left|\vec{k}_{\perp}\right|}{Q} D(y) g_{L}^{\perp} \cos \phi,
$$




$$
\begin{aligned}
W_{L T}= & \frac{\left|\vec{k}_{\perp}\right|}{M} C(y) g_{1 T}^{\perp} \cos \left(\phi-\phi_{s}\right) \\
& -\frac{2 x_{B} M}{Q} D(y)\left[g_{T} \cos \phi_{s}-\frac{k_{\perp}^{2}}{2 M^{2}} g_{T}^{\perp} \cos \left(2 \phi-\phi_{s}\right)\right],
\end{aligned}
$$

where the 1st subscript of $W_{s_{l} s}$ stands for the lepton spin and the 2nd subscript stands for the nucleon spin.

Up to twist- 4 the cross section for unpolarized SIDIS is, ${ }^{6}$

$$
\begin{aligned}
\frac{d \sigma}{d x_{B} d y d^{2} k_{\perp}}= & \frac{2 \pi \alpha_{e m}^{2} e_{q}^{2}}{Q^{2} y}\left\{\left[1+(1-y)^{2}\right] f_{q}^{N}\left(x_{B}, k_{\perp}\right)\right. \\
& -4(2-y) \sqrt{1-y} \frac{\left|\vec{k}_{\perp}\right|}{Q} x_{B} f_{q \perp}^{(1) N}\left(x_{B}, k_{\perp}\right) \cos \phi \\
& -4(1-y) \frac{\left|\vec{k}_{\perp}\right|^{2}}{Q^{2}} x_{B}\left[\varphi_{\perp 2}^{(1) N}\left(x_{B}, k_{\perp}\right)-\tilde{\varphi}_{\perp 2}^{(1) N}\left(x_{B}, k_{\perp}\right)\right] \cos 2 \phi \\
& +8(1-y) \frac{2 x_{B}^{2} M^{2}}{Q^{2}} f_{q(-)}^{N}\left(x_{B}, k_{\perp}\right) \\
& \left.-2\left[1+(1-y)^{2}\right] \frac{\left|\vec{k}_{\perp}\right|^{2}}{Q^{2}} x_{B}\left[\varphi_{\perp}^{(2, L) N}\left(x_{B}, k_{\perp}\right)-\tilde{\varphi}_{\perp}^{(2, L) N}\left(x_{B}, k_{\perp}\right)\right]\right\} .
\end{aligned}
$$

Various azimuthal asymmetries can be obtained from above cross sections, and they are proportional to the ratios of different TMD parton distribtuion/correlation functions. It is of fundamental importance to measure these azimuthal asymmetries to study various functions that describe different aspects of the partonic structure of nucleon.

\section{Nuclear Effects on Azimuthal Asymmetries}

For nucleus involved SIDIS $e+A \rightarrow e+q+X$, the cross sections and azimuthal asymmetries can be obtained with the similar collinear expansion technique as the SIDIS $e+N \rightarrow e+q+X$, but the parton distribution/correlation functions is modified by the nuclear envirenment. Under the assumption of loosely bounded nucleus and taking only maximal nuclear effects,${ }^{10}$ has proposed a method to quantitatively calculate nuclear modifications to parton distribution/correlation funtions. We have systematically applied this method to the study of higher twist parton correlation functions, and obtian the nuclear dependence of azimuthal asymmetries. ${ }^{5-8}$ Further we take identical Gaussian ansatz for all transverse momentum distributions to study the quantitative behaviour of the nuclear dependence. We successfully describe the A-dependence of hadron production cross section, and predict the universal A-dependence of azimuthal asymmetries, as shown in Fig. 2. 


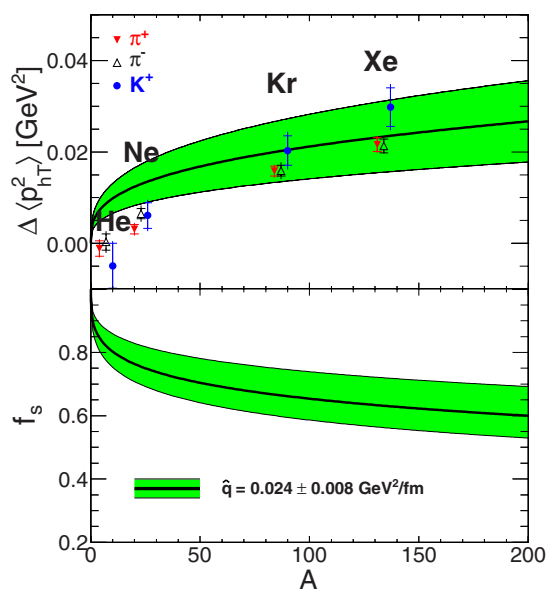

Fig. 2. Upper panel, nuclear dependence of hadron production cross section; lower panel, Nuclear dependence of azimuthal asymmetires. Figure from Ref. 8.

\section{Conclusion}

We have systematically applied the collinear expansion technique to semi-inclusive DIS process, and study their cross section and azimuthal asymmetries for various polarization scenario up to twist-3 and twist-4. We have studied the nuclear effects on azimuthal asymmetries, and predict their A-dependences which will be tested in the future experiments involving nuclear target, such as JLab and EIC.

\section{References}

1. R. K. Ellis, W. Furmanski and R. Petronzio, Nucl. Phys. B207, p. 1 (1982).

2. R. Ellis, W. Furmanski and R. Petronzio, Nucl.Phys. B212, p. 29 (1983).

3. J.-W. Qiu, Phys. Rev. D42, 30 (1990).

4. Z.-t. Liang and X.-N. Wang, Phys. Rev. D75, p. 094002 (2007).

5. J.-H. Gao, Z.-t. Liang and X.-N. Wang, Phys. Rev. C81, p. 065211 (2010).

6. Y.-k. Song, J.-h. Gao, Z.-t. Liang and X.-N. Wang, Phys. Rev. D83, p. 054010 (2011).

7. Y.-k. Song, J.-h. Gao, Z.-t. Liang and X.-N. Wang, Phys.Rev. D89, p. 014005 (2014).

8. Y.-k. Song, Z.-t. Liang and X.-N. Wang, Phys.Rev. D89, p. 117501 (2014).

9. R. N. Cahn, Phys. Lett. B78, p. 269 (1978).

10. Z.-t. Liang, X.-N. Wang and J. Zhou, Phys. Rev. D77, p. 125010 (2008). 